\title{
Experiencia inicial con esofagectomia mínimamente invasiva transtorácica en posición semiprono por cáncer esofágico
}

\author{
Drs. Enrique Norero ${ }^{1}$, Marco Ceroni ${ }^{1}$, Antonio Ramírez ${ }^{1}$, Ricardo Mejía ${ }^{1}$, Cristian Martínez ${ }^{1}$, \\ Rodrigo Muñoz ${ }^{1}$, Fernando Araos ${ }^{1}$, Paulina González ${ }^{1}$ y Alfonso Díaz ${ }^{1}$
}

\section{Initial experience with minimally invasive transtoraxic esophagectomy in semiprone position for esophageal cancer}

Introduction: Surgical treatment of esophageal cancer is associated with high morbidity and mortality. The minimally invasive approach has been introduced with the aim of reducing postoperative morbidity. Aim: To describe the surgical technique and the results of transthoracic minimally invasive esophagectomy (MIE) in semiprone position. Material and Methods: Descriptive cohort study. Patients with an elective MIE for cancer were included between April 2013 and May 2017. Demographic, perioperative, pathology and survival variables were recorded. Results: We included 33 patients $(24$ men, age 69 years, $91 \%$ with comorbidities). The predominant location of the tumor was in the middle and lower thirds of the esophagus (90\%). Fifteen (45\%) patients received neoadjuvant treatment. There were no cases of conversion to thoracotomy. The reconstruction was performed with stomach in $93 \%$. Cervical anastomosis was performed in $66 \%$ and thoracic anastomosis in 30\%. The operative time was $420(330-570)$ minutes and bleeding $200(20-700) \mathrm{cc}$. The 90 -day mortality rate was $0 \%$. Overall morbidity was $78 \%$, there was a $15 \%$ occurrence of pneumonia and $9 \%$ required a reoperation. The hospital stay was 23 (11-81) days. The histology was squamous carcinoma in $51 \%$ and adenocarcinoma in $45 \%$. Margins were RO at $87 \%$. The lymph node count reached 30 (9-45) lymph nodes. Overall 2-year survival is $68 \%$. Conclusion: The preliminary results of this technique are favorable, without any case of postoperative mortality. The oncological results demonstrate a high percentage of RO surgery and adequate lymph node count.

Key words: Esophageal neoplasms, Esophagectomy, Prone position, Minimally invasive surgical procedures.

\section{Resumen}

Introducción: El tratamiento quirúrgico del cáncer esofágico se asocia a una alta morbimortalidad. El abordaje mínimamente invasivo se ha introducido con el objetivo de disminuir la morbilidad postoperatoria. Objetivo: Describir la técnica y los resultados de la esofagectomía mínimamente invasiva (EMI) transtorácica en posición semiprono. Métodos: Estudio de cohorte descriptivo. Se incluyeron pacientes con una EMI electiva por cáncer entre abril de 2013 y mayo de 2017. Se registraron variables demográficas, perioperatorias, anatomía patológica y la sobrevida. Resultados: Incluimos 33 pacientes (24 hombres, edad 69 años, 91\% con comorbilidades). La ubicación predominante del tumor fue en los tercios medio e inferior del esófago (90\%). Quince (45\%) pacientes recibieron neoadyuvancia. No existieron casos de conversión a toracotomías. La reconstrucción se realizó con estómago en un 93\%. Se realizó anastomosis cervical en $66 \%$ y torácica en 30\%. El tiempo operatorio fue de 420 (330-570) minutos y el sangrado de 200 (20-700) cc. La mortalidad a 90 días fue de 0\%. La morbilidad global fue de 78\%, se registró un $15 \%$ de neumonía y un 9\% requirió una reoperación. La estadía hospitalaria fue de 23 (11-81) días. La histología fue carcinoma escamoso en 51\% y adenocarcinoma en 45\%. Los márgenes fueron RO en $87 \%$. El recuento ganglionar alcanzó 30 (9-45) ganglios. La sobrevida global a 2 años es 68\%. Conclusión: Los resultados preliminares de esta técnica son favorables; sin ningún caso de mortalidad postoperatoria. Los resultados oncológicos demuestran un alto porcentaje de cirugía RO y adecuado recuento ganglionar.

Palabras clave: Neoplasias esofágicas, esofagectomía, posición prono, procedimiento quirúrgico mínimamente invasivo.

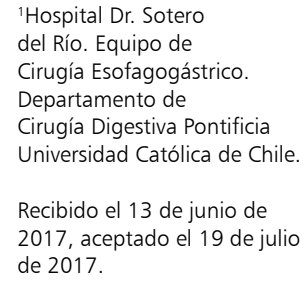

Correspondencia a: Dr. Enrique Norero enorero@uc.cl enorero@yahoo.com 


\section{Introducción}

La esofagectomía es una de las cirugías que se asocia a una de las más altas mortalidades postoperatorias entre procedimientos electivos, llegando al $20 \%$ en centros con bajo volumen de casos y alrededor de $8 \%$ en centro de alto volumen ${ }^{1}$. Destacando un alto porcentaje de complicaciones respiratorias, que superan el $30 \%$ en cirugía abierta ${ }^{2,3}$.

El mejor abordaje en la esofagectomía ha sido discutido por décadas, sin un acuerdo definitivo sobre cuál es el mejor procedimiento ${ }^{4,5}$. El abordaje transtorácico ha demostrado una mejor sobrevida a largo plazo en un grupo de pacientes con enfermedad ganglionar limitada en un estudio randomizado controlado ${ }^{4}$. Sin embargo el abordaje transtorácico tiene una tendencia a mayor morbilidad respiratoria que el abordaje transhiatal ${ }^{4}$.

Con la aparición y el desarrollo de la esofagectomía mínimamente invasiva (EMI), se evita la necesidad de la toracotomía, lo cual reduce el dolor y las complicaciones respiratorias ${ }^{5,6}$. Se han descrito varias alternativas de EMI, entre estas existen distintas posiciones de trabajo en la fase torácica de la cirugía, entre las cuales está la posición clásica en decúbito lateral ${ }^{6}$, la posición prono ${ }^{7}$ y la posición semiprono $^{8}$. Las posiciones prono y semiprono permiten la caída del pulmón hacia anterior logrando un adecuado campo operatorio ${ }^{7,8}$ y la posición semiprono podría tener beneficios al disminuir las dificultades en la monitorización y en el manejo de la vía aérea por el equipo anestésico comparado con la posición prono.

El objetivo del estudio es describir la técnica de EMI transtorácica en posición semiprono y comunicar nuestros resultados preliminares.

\section{Materiales y Métodos}

Se incluyó a los pacientes sometidos a una EMI en posición semiprono en forma consecutiva sin exclusiones realizadas en un centro, desde el 2013 hasta el 2017, debido a cáncer esofágico o de unión gastroesofágica.

La evaluación preoperatoria del paciente consistió en hemograma, perfil bioquímico, pruebas hepáticas y ECG. Se realizó una evaluación nutricional por el equipo de nutrición. Se evaluó el riesgo cardiológico y se realizó un cintigrama miocárdico. La evaluación respiratoria consistió en una espirometría y gases arteriales.

En las semanas previas a la EMI los pacientes fueron evaluados por el equipo de kinesioterapia.
La evaluación del tumor consistió en una endoscopia y biopsia. En todos los pacientes se realizó un TC de cuello, tórax, abdomen y pelvis, y en pacientes con cáncer clínicamente T2 o superior, o según los hallazgos del TC se recomendó la realización de un PET-CT. Se utilizó endosonografía (EUS) en tumores en que el endosonógrafo podía ser avanzado a distal del tumor. En tumores de tercio medio y superior se realizó una fibrobroncoscopia para descartar compromiso de la vía aérea.

Todos los casos se discutieron en reunión del equipo de cirugía y en comité oncológico. Se indicó tratamiento neoadyuvante en pacientes menores de 75 años, con buena función cardiológica, renal y hematológica, que contaban con una vía de alimentación apropiada, clínicamente T3 o con ganglios positivos. Se eligió tratamiento con radioterapia-quimioterapia en carcinoma escamoso ${ }^{9}$ y quimioterapia exclusiva para pacientes con adenocarcinoma ${ }^{10}$.

\section{Técnica quirúrgica}

Se utiliza tubo orotraqueal de doble lumen izquierdo, catéter venoso central, lineal arterial, sonda Foley y sonda nasogástrica. Se coloca al paciente en posición semiprono (Figura 1), logrando un ángulo de $50-60^{\circ}$ entre la mesa operatoria y el tórax del paciente.

Se instalan 3 trocares de $10-12 \mathrm{~mm}$ posteriores a la altura de la línea axilar posterior y un trocar de $5 \mathrm{~mm}$ en posición media entre línea axilar posterior y la columna, en el hemitórax derecho. Se utiliza óptica de $30^{\circ}$. Se usa $\mathrm{CO}_{2}$ con una presión de 8 $\mathrm{mmHg}$. Se inicia la disección abriendo la pleura mediastínica. A continuación se diseca el arco de la vena ácigos, el cual se secciona con sutura lineal o con tijera luego de colocar clips. Se diseca hacia superior, separando el esófago de la columna dorsal y de la vía aérea; y hacia el mediastino inferior separándolo del pericardio, aorta y pleura izquierda. Se incluye en la disección los ganglios periesofágicos e infracarinales (Figura 1).

En la fase abdominal, según la evolución de la serie, se realiza una laparotomía o una laparoscopia con 5-6 trocares en el abdomen superior. Se disecan los pilares y el tronco celiaco y sus ramas. Se colocan clips y seccionan los vasos gástricos izquierdos por separado. Se realiza la liberación del estómago seccionando los vasos gástricos cortos y se secciona el ligamento gastrocólico respetando la arcada gastroepiploica. Se diseca y secciona el esófago a nivel cervical y se exterioriza la pieza quirúrgica por el abdomen. Se prepara un tubo gástrico de $4 \mathrm{~cm}$ de ancho con disparos secuenciales de sutura mecánica de 3,5 $\mathrm{mm}$ de altura con posterior invaginación de 


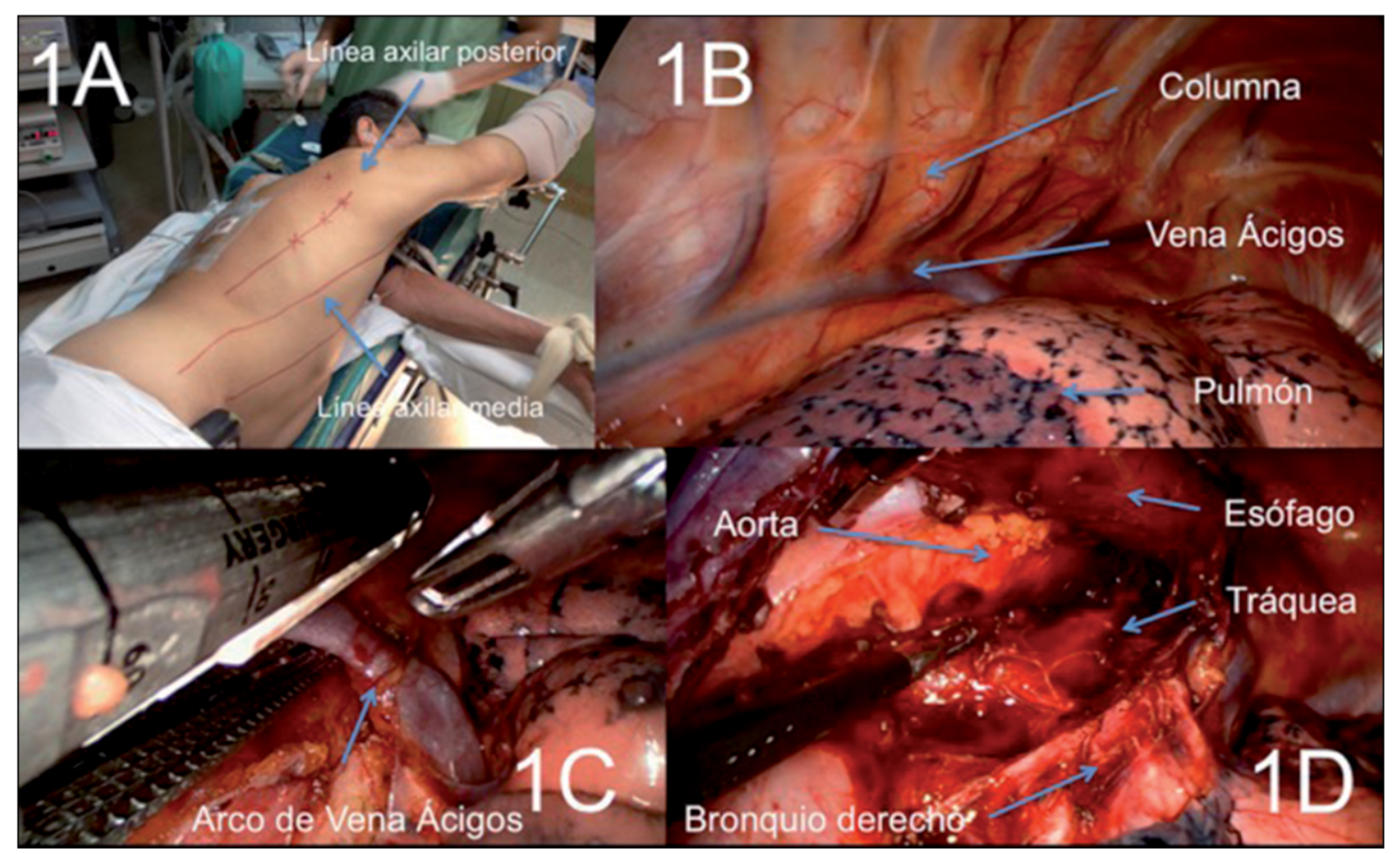

Figura 1. Posición del paciente y disección transtorácica. A: Posición semiprono del paciente con cojín y soporte, y posición de trocares en la línea axilar posterior. B: Videotoracoscopia con el pulmón desplazado hacia inferior por gravedad y visión del mediastino posterior. C: Sección del arco de la vena ácigos con sutura mecánica. D: Disección del esófago, separado de la vía aérea y la aorta. la línea de sutura con monofilamento 3.0. Se realiza una yeyunostomía. Se asciende al estómago por mediastino posterior y se realiza una anastomosis esofago-gástrica a nivel cervical, en la mayoría de los casos con sutura circular mecánica.

Luego de los primeros 10 casos realizados por laparotomía cambiamos a un abordaje laparoscópica en la fase abdominal y realizamos solo una mini laparotomía de $4 \mathrm{~cm}$ para exteriorizar la pieza. En la segunda mitad de la serie, en pacientes con tumores de tercio inferior, realizamos una EMI tipo Ivor-Lewis, partiendo por laparoscopia y luego el tiempo torácico con anastomosis torácica con sutura mecánica circular (Figura 2).

Se utilizó la definición de complicaciones publicada según el consenso internacional de esofagectomía, a 90 días de la cirugía ${ }^{11}$ y se graduó las complicaciones según la clasificación de Clavien ${ }^{12}$. Se incluyeron en la evaluación las complicaciones alejadas tales como estenosis de la anastomosis y hernia hiatal, las que se consideraron durante todo el seguimiento de los pacientes.

Se utilizó el TNM según la séptima edición ${ }^{13}$. Se realizó seguimiento en policlínico y periódicamente un TC para evaluar recidiva.

Se utilizó el programa SPSS y minitab. Se realizó curva de sobrevida global según Kaplan-Meier. El comité de ética aprobó la realización del estudio.

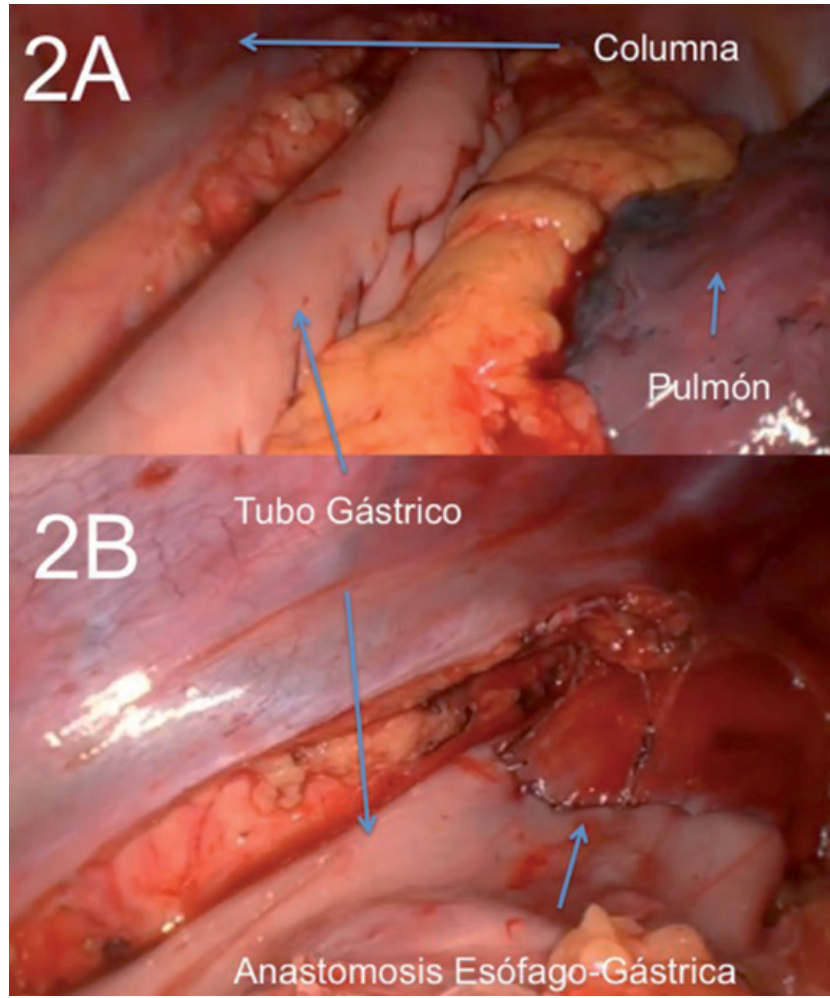

Figura 2. Reconstrucción con tubo gástrico. A: Visión del tubo gástrico en el mediastino posterior. B: Anastomosis esofagogástrica en el mediastino posterior alto. 


\section{Resultados}

Se incluyeron en el estudio 33 pacientes (hombres 24; edad 69 [41-78] años; IMC 24 [17-32]) que se sometieron a una EMI, lo cual representa el $78 \%$ de las esofagectomías realizadas por cáncer esofágico en el período de estudio (Tabla 1).

Treinta $(91 \%)$ pacientes presentaban alguna comorbilidad, dentro de estos un $12 \%$ se consideraron ASA 3. En $15(45 \%)$ pacientes se indicó aporte nutricional enteral previo a la cirugía, mediante una gastrostomía en 2, sonda nasogástrica en 3 y una yeyunostomía en 10 pacientes (Tabla 1).

La ubicación tumoral más frecuente fue el tercio inferior del esófago en 20 pacientes $(60 \%)$ y la mediana de longitud tumoral fue de $4(2-8) \mathrm{cm}$. La etapificación se realizó con PET-CT en un $57 \%$ de los pacientes (Tabla 1). En 15 (45\%) pacientes se realizó neoadyuvancia; en 9 con radioquimioterapia $\mathrm{y}$ en 6 con quimioterapia.

En un $69 \%$ de los pacientes se realizó una EMI tipo McKeown con un tiempo torácico inicial, a continuación un tiempo abdominal y una anastomosis cervical; y en un $30 \%$ se realiza una EMI tipo Ivor-Lewis con un tiempo laparoscópico, a continuación el tiempo torácico y una anastomosis torácica. No tuvimos casos de conversión en la fase torácica y solo $1(3 \%)$ caso de conversión en el tiempo laparoscópico debido a adherencias de cirugías previas. La reconstrucción se realizó con estómago en un $93 \%$ y en 1 paciente no se realizó debido a una lesión compleja de la vía aérea asociado a la instalación del tubo orotraqueal. El sangrado operatorio fue de $200 \mathrm{cc}(20-700)$ y el tiempo operatorio total fue de $420 \mathrm{~min}$ (330-570) (Tabla 2).

La mortalidad operatoria a 90 días fue de $0 \%$. La morbilidad global fue de $78 \%$. Las complicaciones más frecuentes fueron la filtración de la anastomosis esofago-gástrica cervical en un $63 \%$, las complicaciones respiratorias en un $24 \%$ y la retención gástrica en un $21 \%$. Tres pacientes requirieron una reoperación, el primero debido a una filtración de una anastomosis cervical que se dirigió al mediastino y a la pleura derecha, realizándose aseo y desfuncionalización por toracotomía; en un segundo paciente se ligó el conducto torácico por videotoracoscopia debido a una fístula quilosa de alto volumen, y en un tercer paciente con una filtración torácica se realizó un aseo por videotorascoscopia. Siete $(21 \%)$ pacientes requirieron estadía en la unidad de cuidados intensivos, 2 de ellos en el postoperatorio inmediato debido a una cirugía prolongada, 2 debido a falla respiratoria, 2 pacientes reoperados debido a filtración y uno para monitorización debido a neumonía
Tabla 1. Características preoperatorias de los pacientes y del tumor esofágico

\begin{tabular}{|c|c|}
\hline Sexo masculino & $24(72,7 \%)$ \\
\hline Edad (años) & $69(41-78)$ \\
\hline $\mathrm{IMC}\left(\mathrm{kg} / \mathrm{mt}^{2}\right)$ & $24(17-32)$ \\
\hline $\begin{array}{l}\text { Comorbilidades } \\
\text { Hipertensión } \\
\text { Diabetes } \\
\text { EPOC } \\
\text { DHC } \\
\text { Cardiopatía } \\
\text { RGE } \\
\text { Esófago de Barrett }\end{array}$ & $\begin{array}{rc}19 & (57,6 \%) \\
8 & (24,2 \%) \\
1 & (3,0 \%) \\
2 & (6,1 \%) \\
3 & (9,1 \%) \\
6 & (18,2 \%) \\
3 & (9,0 \%)\end{array}$ \\
\hline $\begin{array}{l}\text { Puntaje de ASA } \\
\quad \text { I } \\
\text { II } \\
\text { III }\end{array}$ & $\begin{array}{r}3(9,0 \%) \\
26(78,7 \%) \\
4(12,1 \%)\end{array}$ \\
\hline Cirugía abdominal previa & $14(42,4 \%)$ \\
\hline Cirugía torácica previa & $1 \quad(3,0 \%)$ \\
\hline Consumo de tabaco & $16(48,4 \%)$ \\
\hline Consumo de alcohol & $13(39,3 \%)$ \\
\hline Albúmina (g/dl) & $4,1(2,8-4,6)$ \\
\hline Prealbúmina (g/dl) & $22(8-35)$ \\
\hline $\mathrm{VEF}_{1}(\%)$ & $112(77-171)$ \\
\hline CVF (\%) & $119(73-175)$ \\
\hline Alimentación enteral pre-op & $15(45,4 \%)$ \\
\hline $\begin{array}{l}\text { Ubicación del tumor } \\
\text { Tercio superior } \\
\text { Tercio medio } \\
\text { Tercio inferior }\end{array}$ & $\begin{array}{c}3(9,1 \%) \\
10(30,3 \%) \\
20(60,6 \%)\end{array}$ \\
\hline Compromiso de UGE & $9(27 \%)$ \\
\hline Largo del tumor $(\mathrm{cm})$ & $4(2-8)$ \\
\hline Etapificación con TC & $33(100 \%)$ \\
\hline Etapificación con PET-CT & $19(57,5 \%)$ \\
\hline
\end{tabular}

IMC: índice de masa corporal. UGE: Unión gastroesofágica. $V_{E F}$ : volumen espiratorio forzado. CVF: Capacidad vital forzada. EPOC: enfermedad pulmonar obstructiva crónica. DHC: daño hepático crónico. RGE: reflujo gastroesofágico. UGE: unión gastroesofágica.

sin necesidad de ventilación mecánica; los restantes $26(78 \%)$ pacientes fueron extubados al término de la cirugía y se trasladaron desde la unidad de recuperación al servicio de cirugía entre el primer y segundo día postoperatorio, sin requerimiento de UCI durante toda su hospitalización. La mediana de estadía hospitalaria fue de 23 (11-81) días (Tabla 2).

En el seguimiento, 5 (15\%) pacientes evolucio- 
Tabla 2. Información de esofagectomía mínimamente invasiva y complicaciones postoperatorias

\begin{tabular}{|c|c|}
\hline $\begin{array}{l}\text { Técnica de esofagectomía } \\
\text { McKeown } \\
\text { Ivor Lewis }\end{array}$ & $\begin{array}{ll}23 & (69 \%) \\
10 & (30,3 \%)\end{array}$ \\
\hline Fase torácica por videotoracoscopia & $33(100 \%)$ \\
\hline Conversión videotoracoscopia & $0 \quad(0 \%)$ \\
\hline Fase abdominal laparoscópica & $22(66,7 \%)$ \\
\hline Conversión laparoscopia & $1 \quad(3 \%)$ \\
\hline $\begin{array}{l}\text { Reconstrucción } \\
\text { Estómago } \\
\text { Colon } \\
\text { Sin reconstrucción }\end{array}$ & $\begin{aligned} 31 & (93,9 \%) \\
1 & (3 \%) \\
1 & (3 \%)\end{aligned}$ \\
\hline Sangrado operatorio (cc) & $200(20-700)$ \\
\hline Tiempo operatorio total (min) & $420(330-570)$ \\
\hline Mortalidad operatoria & $0 \quad(0 \%)$ \\
\hline Morbilidad operatoria & $26(78,7 \%)$ \\
\hline Reoperación & $3 \quad(9,1 \%)$ \\
\hline Estadía en UCI* & $7(21,2 \%)$ \\
\hline Fístula anastomosis cervical & $14(63 \%)$ \\
\hline Fístula anastomosis torácica & $2(20 \%)$ \\
\hline Estenosis anastomosis cervical & $5(15 \%)$ \\
\hline Estenosis anastomosis torácica & $0 \quad(0 \%)$ \\
\hline $\begin{array}{l}\text { Respiratorias } \\
\text { Neumonía } \\
\text { Derrame pleural } \\
\text { Falla respiratoria (VM) } \\
\text { Lesión vía aérea }\end{array}$ & $\begin{array}{ll}8 & (24,2 \%) \\
5 & (15,2 \%) \\
3 & (9,1 \%) \\
2 & (6,1 \%) \\
1 & (3 \%)\end{array}$ \\
\hline Retención gástrica & $7(21,2 \%)$ \\
\hline Paresia de cuerda vocal & $2 \quad(6,1 \%)$ \\
\hline Quilotórax & $2 \quad(6,1 \%)$ \\
\hline Fibrilación auricular & $2 \quad(6,1 \%)$ \\
\hline Sepsis por catéter & $(6,1 \%)$ \\
\hline Colitis por Clostridium & $2 \quad(6,1 \%)$ \\
\hline Falla renal aguda & $3 \quad(9,0 \%)$ \\
\hline Embolia pulmonar & $2 \quad(6,1 \%)$ \\
\hline Hernia hiatal & $2 \quad(6,1 \%)$ \\
\hline $\begin{array}{l}\text { Clavien } \\
\text { I } \\
\text { II } \\
\text { III } \\
\text { Illb } \\
\text { IVa } \\
\text { IVb }\end{array}$ & $\begin{aligned} 7 & (21,2 \%) \\
12 & (36,4 \%) \\
2 & (6,1 \%) \\
1 & (3 \%) \\
2 & (6,1 \%) \\
2 & (6,1 \%)\end{aligned}$ \\
\hline Estadía hospitalaria (días) & $23(11-81)$ \\
\hline
\end{tabular}

*En postoperatorio inmediato o debido a complicaciones. McKeown: esofagectomía con tiempo torácico inicial y anastomosis cervical. Ivor Lewis: esofagectomía con tiempo abdominal inicial y anastomosis torácica. naron con una estenosis de la anastomosis esofagogástrica cervical y se trataron por vía endoscópica exitosamente. Dos $(6,1 \%)$ pacientes presentaron hernia hiatal con ascenso del colon transverso a través del hiato esofágico, ambos fueron diagnosticados y operados a los 12 meses de la EMI, uno de ellos por vía laparoscópica y otro por laparotomía.

La histología de los pacientes operados fue carcinoma escamoso en 17 (51\%), adenocarcinoma en $15(45 \%)$ y adenoescamoso en un caso $(3 \%)$. Se logró el RO en 29 (87\%) pacientes, en 2 casos (6\%) el margen radial fue microscópicamente positivo, en otro caso $(3 \%)$ se diagnosticó intraoperatoriamente infiltración de la tráquea y la cirugía fue R2, un cuarto $(3 \%)$ paciente se encontraba en etapa IV con una metástasis hepática única al momento de la cirugía. La mediana de ganglios resecados fue de 30 (9-45) ganglios; 9 (3-26) en el tórax y 19 (5-32) en el abdomen. Los pacientes sometidos a neoadyuvancia se consideraron todos clínicamente T3 y en 9 de ellos se consideraron clínicamente con ganglios positivos. Seis de los pacientes tratados con neoadyuvancia presentaron respuesta patológica completa (yTONOMO). Los pacientes sometidos a cirugía exclusiva se encontraban en etapa I-II en 10 casos y en etapa III en 8 casos (Tabla 3).

La mediana de seguimiento fue de 16 (1-43) meses. $26(79 \%)$ pacientes se encuentran vivos y 7 (21\%) han fallecido debido a cáncer esofágico. La sobrevida global a 2 años es de $68 \%$ (mediana no alcanzada) (Figura 3).

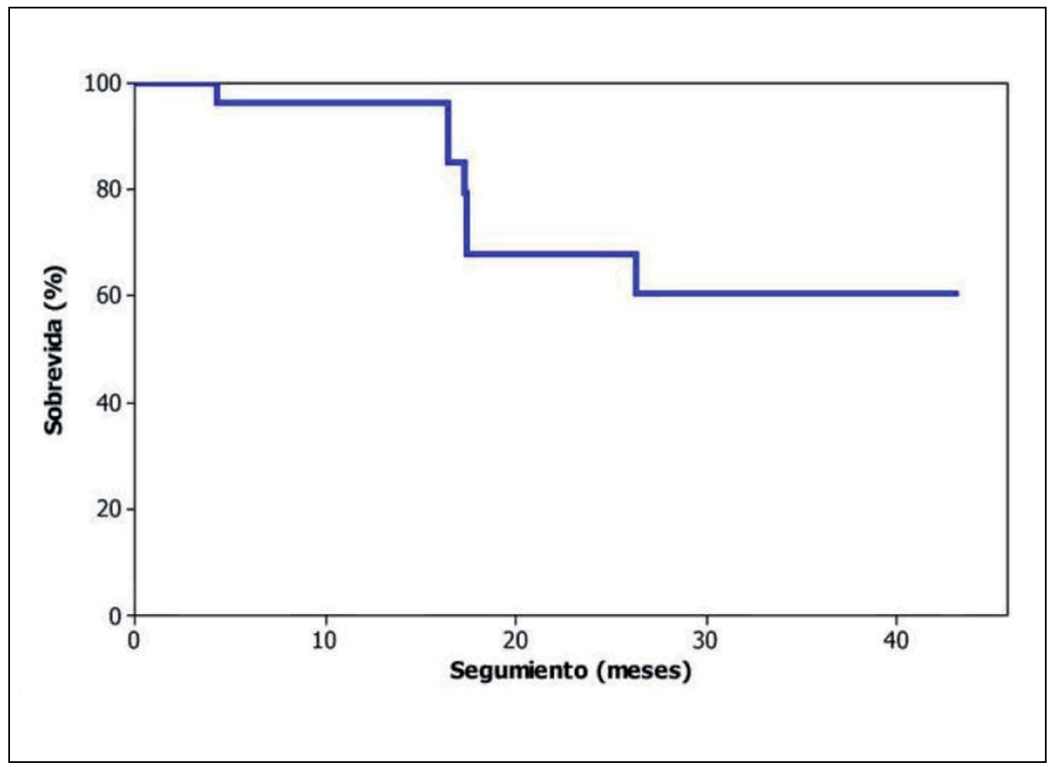

Figura 3. Sobrevida global de pacientes sometidos a esofagectomía mínimamente invasiva. 
Tabla 3. Etapificación clínica y patológica en pacientes con neoadyuvancia $(3 \mathrm{~A})$ y con cirugía exclusiva (3B)

A. Pacientes sometidos a neoadyuvancia $(n=15)$

\begin{tabular}{|c|c|}
\hline \multicolumn{2}{|l|}{ Clínica } \\
\hline $\mathrm{Tl}$ & $0 \quad(0 \%)$ \\
\hline $\mathrm{T} 2$ & $0 \quad(0 \%)$ \\
\hline $\mathrm{T} 3$ & $15(45 \%)$ \\
\hline $\mathrm{T} 4$ & $0 \quad(0 \%)$ \\
\hline $\mathrm{N}$ positivo & $9(27 \%)$ \\
\hline M positivo & $1(3 \%)$ \\
\hline \multicolumn{2}{|l|}{ Patológica } \\
\hline yTO & $6(18 \%)$ \\
\hline yTl & $3(9 \%)$ \\
\hline yT2 & $2(6 \%)$ \\
\hline yT3 & $4(12 \%)$ \\
\hline yNO & $11(33 \%)$ \\
\hline yNl & $2(6 \%)$ \\
\hline $\mathrm{yN} 2$ & $1 \quad(3 \%)$ \\
\hline yN3 & $1 \quad(3 \%)$ \\
\hline yEtapa 0 & $6(18 \%)$ \\
\hline yEtapa I & $4(12 \%)$ \\
\hline yEtapa II & $2(6 \%)$ \\
\hline yEtapa III & $2(6 \%)$ \\
\hline yEtapa IV & $1 \quad(3 \%)$ \\
\hline
\end{tabular}

B. Pacientes con cirugía exclusiva $(n=18)$

\begin{tabular}{|l|c|}
\hline T1 & $7(21 \%)$ \\
T2 & $3(9 \%)$ \\
T3 & $7(21 \%)$ \\
T4 & $1(3 \%)$ \\
N0 & $9(27 \%)$ \\
N1 & $4(12 \%)$ \\
N2 & $3(9 \%)$ \\
N3 & $2(6 \%)$ \\
Etapa I & $8(24 \%)$ \\
Etapa II & $2(6 \%)$ \\
Etapa III & $8(24 \%)$ \\
Etapa IV & $0(0 \%)$ \\
\hline
\end{tabular}

\section{Discusión}

En Chile el cáncer de esófago es poco frecuente ${ }^{14}$, y por otro lado, la mortalidad de la esofagectomía es alta ${ }^{1,2}$. Estas son algunas de las razones por las cuales se realizan un número reducido de esofagectomías en nuestro país, el que se estima en 63 al año; la experiencia nacional reportada viene de algunos centros específicos ${ }^{14,16,17}$.
Durante décadas se ha discutido cuál es el mejor abordaje quirúrgico del cáncer de esófago ${ }^{4,5}$. Desde la aparición de la EMI, se ha producido un interés exponencial en su desarrollo, ya que permite una esofagectomía transtorácica, evitando la morbilidad asociada de la toracotomía ${ }^{6,7}$. Actualmente, estudios randomizados controlados y metaanálisis apoyan su mejor resultado perioperatorio comparado con la cirugía abierta, además, de resultados oncológicamente equivalentes en el largo plazo ${ }^{3,18,19}$.

A partir de la experiencia previa en cirugía abierta de esófago y del desarrollo de la cirugía mínimamente invasiva en patología oncológica digestiva en nuestro centro, hemos desarrollado nuestra técnica de EMI. Los resultados presentados en esta publicación inicial demuestran $0 \%$ de conversión a toracotomía y $0 \%$ de mortalidad postoperatoria a 90 días, si bien el número de pacientes es aún pequeño, representa 4 años de EMI sin mortalidad asociada.

Sin embargo, la morbilidad es alta y está presente en el $78 \%$ de los pacientes. Dentro de las principales causas de mortalidad operatoria postesofagectomía destacan las complicaciones respiratorias $^{2}$. Un reciente estudio randomizado controlado describe una disminución significativa en neumonía postoperatoria de $34 \%$ en esofagectomía abierta a un $12 \%$ en $\mathrm{EMI}^{3}$, lo que es comparable a nuestro resultado de $15 \%$ de neumonía postoperatoria y creemos que se asocia al abordaje mínimamente invasivo. La complicación quirúrgica más frecuente fue la filtración de la anastomosis esofagogástrica, la cual fue mayor para la anastomosis cervical que la anastomosis torácica, tal como se ha reportado en un metaanálisis de estudios randomizados controlados ${ }^{21}$. Otro hallazgo interesante de nuestro estudio, es que el $78 \%$ de los pacientes no requirieron estadía en UCI en ningún momento de su evolución postoperatoria. En la mayoría de centros, se describe como parte del postoperatorio normal la estadía en UCI al menos los primeros días ${ }^{4,6}$. Creemos que esto se debe en parte al menor impacto fisiológico de la cirugía mínimamente invasiva y a que solo en 7 pacientes se presentó una complicación grave.

El RO es de $87 \%$ y el recuento ganglionar alcanza los 30 ganglios, también comparable a series de esofagectomía abierta y laparoscópica ${ }^{3,7,16,22,23}$, lo cual apoya la buena visión y el adecuado campo de trabajo que permite la EMI en posición semiprono. Por otro lado, el uso de la neoadyuvancia no es una limitación para realizar EMI, ya que en nuestro estudio se utilizó en un $45 \%$. Al contrario, apoyado en estudios previos ${ }^{9,10}$, creemos que su uso favorece el alto porcentaje de cirugías con 
márgenes negativos que logramos. El tiempo de seguimiento es muy corto por lo que la evaluación de sobrevida requiere un mayor plazo y se evaluará en los próximos años.

Es difícil la comparación de la posición durante una $\mathrm{EMI}^{8}$. En la literatura, con algunos estudios retrospectivos, un estudio randomizado controlado y metaanálisis, se describe un menor tiempo operatorio, mejor ergonomía, menores complicaciones, menor estadía y mayor número de ganglios resecados en posición prono comparado a posición lateral ${ }^{24,28}$. La técnica semiprono descrita en nuestro estudio, se asemeja más a la posición prono que la posición lateral, de hecho en un reciente metaanálisis la posición semiprono se incluye en el grupo de cirugía en posición prono ${ }^{27}$, ya que en prono y semiprono el pulmón cae por gravedad hacia anterior en el tórax y no es necesario la constante separación del pulmón y la aspiración de líquido en el mediastino posterior. Creemos que existen dos ventajas en la posición semiprono, en comparación con la posición prono, la menor dificultad en la monitorización y en el manejo de la vía aérea por el equipo anestésico y la posibilidad de conversión en caso que sea necesario.

Estas ventajas descritas se deben estudiar prospectivamente ya que no existen estudios comparativos entre ambas posiciones.

\section{Limitaciones}

Se trata de un estudio preliminar, con una casuística aún reducida y no hay un grupo control para comparación con cirugía abierta ni con posición lateral o prono en la fase torácica.

\section{Conclusiones}

En este estudio, los resultados preliminares de la esofagectomía mínimamente invasiva transtorácica en posición semiprono son favorables representando una técnica segura, sin mortalidad postoperatoria, un alto porcentaje de cirugía RO y adecuado recuento ganglionar.

\section{Responsabilidades éticas}

Protección de personas y animales. Los autores declaran que para esta investigación no se han realizado experimentos en seres humanos ni en animales.

Confidencialidad de los datos. Los autores declaran que han seguido los protocolos de su centro de trabajo sobre la publicación de datos de pacientes.

Derecho a la privacidad y consentimiento informado. Los autores han obtenido el consentimiento informado de los pacientes y/o sujetos referidos en el artículo. Este documento obra en poder del autor de correspondencia.

\section{Financiación}

División de Cirugía Pontificia Universidad Católica de Chile.

\section{Conflicto de intereses}

Los autores declaran no tener ningún conflicto de intereses.

\section{Bibliografía}

1. Birkmeyer JD, Siewers AE, Finlayson EV, Stukel TA, Lucas FL, Batista I, et al. Hospital volume and surgical mortality in the United States. N Engl J Med. 2002;346(15):1128-37.

2. Ghaferi AA, Birkmeyer JD, Dimick JB. Complications, failure to rescue, and mortality with major inpatient surgery in medicare patients. Ann Surg. 2009;250(6):1029-34.

3. Biere SS, van Berge Henegouwen MI, Maas KW, Bonavina L, Rosman C, García JR, et al. Minimally invasive versus open oesophagectomy for patients with oesophageal cancer: a multicentre, open-label, randomised controlled trial.
Lancet. 2012;379(9829):1887-92.

4. Omloo JM, Lagarde SM, Hulscher JB, Reitsma JB, Fockens P, van Dekken H, et al. Extended transthoracic resection compared with limited transhiatal resection for adenocarcinoma of the mid/ distal esophagus: five-year survival of a randomized clinical trial. Ann Surg. 2007;246(6):992-1000; discussion -1.

5. Hulscher JB, van Sandick JW, de Boer AG, Wijnhoven BP, Tijssen JG, Fockens $P$, et al. Extended transthoracic resection compared with limited transhiatal resection for adenocarcinoma of the esophagus. N Engl J Med. 2002;347(21):1662-9.

6. Luketich JD, Pennathur A, Awais O, Levy RM, Keeley S, Shende M, et al. Outcomes after minimally invasive esophagectomy: review of over 1000 patients. Ann Surg. 2012;256(1):95-103.

7. Palanivelu C, Prakash A, Senthilkumar R, Senthilnathan P, Parthasarathi R, Rajan PS, et al. Minimally invasive esophagectomy: thoracoscopic mobilization of the esophagus and mediastinal lymphadenectomy in prone position-experience of 130 patients. J Am Coll Surg. 2006;203(1):7-16.

8. Lin J, Kang M, Chen C, Lin R. Thoracoscopic oesophageal mobilization during thoracolaparoscopy three-stage oesophagectomy: a comparison of lateral decubitus versus semiprone positions. Interact Cardiovasc Thorac Surg. 2013;17(5):829-34. 
9. van Hagen P, Hulshof MC, van Lanschot JJ, Steyerberg EW, van Berge Henegouwen MI, Wijnhoven BP, et al. Preoperative chemoradiotherapy for esophageal or junctional cancer. N Engl J Med. 2012;366(22):2074-84

10. Ychou M, Boige V, Pignon JP, Conroy T, Bouché $\mathrm{O}$, Lebreton $\mathrm{G}$, et al. Perioperative chemotherapy compared with surgery alone for resectable gastroesophageal adenocarcinoma: an FNCLCC and FFCD multicenter phase III trial. J Clin Oncol. 2011;29(13):1715-21.

11. Low DE, Alderson D, Cecconello I, Chang AC, Darling GE, D'Journo $\mathrm{XB}$, et al. International Consensus on Standardization of Data Collection for Complications Associated With Esophagectomy: Esophagectomy Complications Consensus Group (ECCG). Ann Surg. 2015;262(2):286-94.

12. Clavien PA, Barkun J, de Oliveira ML, Vauthey JN, Dindo D, Schulick RD, et al. The Clavien-Dindo classification of surgical complications: five-year experience. Ann Surg. 2009;250(2):18796.

13. Talsma K, van Hagen $P$, Grotenhuis BA, Steyerberg EW, Tilanus HW, van Lanschot JJ, et al. Comparison of the 6th and 7th Editions of the UICC-AJCC TNM Classification for Esophageal Cancer. Ann Surg Oncol. 2012;19(7):2142-8.

14. Braghetto I, Csendes A, Cornejo A, Amat J, Cardemil G, Burdiles P, et al. Survival of patients with esophageal cancer subjected to total thoracic esophagectomy. Rev Med Chile 2000;128(1):64-74.

15. Csendes A, González G. Rates of digestive surgery in Chile during 2004 and
2005. Analysis of hospital discharge data, excluding colorectal interventions. Rev Chilena de Cirugía. 2008;60(5):379-86.

16. Venturelli A, Soto S, Díaz J, Cardemil B, Sánchez A, Jiménez L. Cáncer de esófago, tratamiento en el Hospital Clínico Regional de Valdivia durante el período 1982-2001. Rev Chil Cir. 2003;55(4):3814.

17. Braghetto I, Cardemil G, Mandioca C, Masia G, Gattini F. Impact of minimally invasive surgery in the treatment of esophageal cancer. Arq Bras Cir Dig 2014;27(4):237-42.

18. Dantoc MM, Cox MR, Eslick GD. Does minimally invasive esophagectomy (MIE) provide for comparable oncologic outcomes to open techniques? A systematic review. J Gastrointest Surg. 2012;16(3):486-94.

19. Watanabe M, Baba Y, Nagai Y, Baba H. Minimally invasive esophagectomy for esophageal cancer: an updated review. Surg Today. 2013;43(3):237-44.

20. Norero E, Báez S, Briceño E, Martínez C, Ceroni M, Escalona A, et al. Totally laparoscopic gastrectomy for the treatment of gastric tumors. Rev Med Chile 2015;143(3):281-8.

21. Biere SS, Maas KW, Cuesta MA, van der Peet DL. Cervical or thoracic anastomosis after esophagectomy for cancer: a systematic review and meta-analysis. Dig Surg. 2011;28(1):29-35.

22. Portale G, Hagen JA, Peters JH, Chan LS, DeMeester SR, Gandamihardja TA, et al. Modern 5-year survival of resectable esophageal adenocarcinoma: single institution experience with 263 patients. J Am Coll Surg. 2006;202(4):588-96; discussion 96-8.
23. Swanson SJ, Batirel HF, Bueno R, Jaklitsch MT, Lukanich JM, Allred E, et al. Transthoracic esophagectomy with radical mediastinal and abdominal lymph node dissection and cervical esophagogastrostomy for esophageal carcinoma. Ann Thorac Surg. 2001;72(6):1918-24; discussion 24-5.

24. Fabian T, Martin J, Katigbak M, McKelvey AA, Federico JA. Thoracoscopic esophageal mobilization during minimally invasive esophagectomy: a headto-head comparison of prone versus decubitus positions. Surg Endosc. 2008;22(11):2485-91.

25. Feng M, Shen $Y$, Wang H, Tan L, Zhang Y, Khan MA, et al.

Thoracolaparoscopic esophagectomy: is the prone position a safe alternative to the decubitus position? J Am Coll Surg. 2012;214(5):838-44.

26. Noshiro H, Iwasaki H, Kobayashi K, Uchiyama A, Miyasaka Y, Masatsugu T, et al. Lymphadenectomy along the left recurrent laryngeal nerve by a minimally invasive esophagectomy in the prone position for thoracic esophageal cancer. Surg Endosc. 2010;24(12):2965-73.

27. Markar SR, Wiggins T, Antonowicz S, Zacharakis E, Hanna GB. Minimally invasive esophagectomy: Lateral decubitus vs. prone positioning; systematic review and pooled analysis. Surg Oncol. 2015;24(3):212-9.

28. Shen Y, Feng M, Tan L, Wang H, Li J, Xi $\mathrm{Y}$, et al. Thoracoscopic esophagectomy in prone versus decubitus position: ergonomic evaluation from a randomized and controlled study. Ann Thorac Surg. 2014;98(3):1072-8. 